\title{
Web navigation for blind users
}

\author{
M. Zajicek ${ }^{\mathrm{a}}$, C. Powell ${ }^{\mathrm{a}}$, C. Reeves ${ }^{\mathrm{b}}$ \\ ${ }^{a}$ Oxford Brookes University, Gipsy Lane, Oxford, OX3 OBP, UK \\ ${ }^{b}$ Royal National Institute for the Blind, 224, Great Portland Street, London, WIN 6AA, UK
}

\section{INTRODUCTION}

The aim of our work is to make the wealth of information on the World Wide Web more readily available to blind people. They must be able to search efficiently for relevant information and make quick and effective decisions about the usefulness of pages they retrieve. We have built a prototype application called BrookesTalk [1] which we believe addresses this need more fully than other speech output Web browsers. Word frequency algorithms are used to provide a set of complementary 'views' of a Web page which help blind people make decisions about its usefulness.

This paper reviews the functionality of BrookesTalk and discusses the evaluative feedback received from blind users at the Royal National Institute for the Blind (RNIB).

\section{WHY A NAVIGATION TOOL?}

Screen readers such as JAWS and browsers such as pwWebSpeak( $\left.{ }^{\mathrm{TM}}\right)$, enable the blind user to move around the screen, jump to interaction objects in the screen and read particular areas of text. These become unwieldy when moving quickly around the Web searching for information and making hasty decisions about which pages or bits of pages might be useful.

BrookesTalk supports users in this activity where speedy decisions are paramount. The functionality of BrookesTalk is based on the activity of sighted users when rapidly assimilating a page i.e. look at headings, links and graphical objects and then scanning text.

BrookesTalk also provides facilities for keeping track of interesting pages or sentences using a scratch pad.

\section{FINDING KEYWORDS AND TRIGRAMS}

A list of keywords for each page are computed based on frequency. Table 1. shows examples of keyword extractions for two different Web pages, from which it can be seen that the small number of keywords could be used to augment or replace information given in the title if it was not available or perhaps not informative.

TABLE 1

\begin{tabular}{|l|l|l|l|}
\hline \multicolumn{1}{|c|}{ Document Title } & $\begin{array}{c}\text { Total No. of } \\
\text { Words }\end{array}$ & \multicolumn{1}{|c|}{ Keywords } & Document Subject \\
\hline $\begin{array}{l}\text { Thinking of getting a } \\
\text { border collie? }\end{array}$ & 1174 & $\begin{array}{l}\text { border, collie, people, collies, } \\
\text { dog, exercise dogs }\end{array}$ & $\begin{array}{l}\text { Information for prospective } \\
\text { border collie owners }\end{array}$ \\
\hline $\begin{array}{l}\text { Comet Hale-Bopp Hope } \\
\text { Page (JPL) }\end{array}$ & 772 & $\begin{array}{l}\text { comet, hale, image, January, } \\
\text { images, vodniza }\end{array}$ & $\begin{array}{l}\text { Information about comet } \\
\text { Hale-Bopp }\end{array}$ \\
\hline
\end{tabular}

When keywords are used all contextual information imparted by the position of a word in a sentence is lost. Extraction of three word key phrases or trigrams preserves some word position information. To provide a measure of similarity, groups of words appearing together rather than individual words are compared. 
The trigram module in BrookesTalk takes the printable text of the document and from it constructs the set of word level trigrams. To reduce the number of word-level mismatches due to the normal changes in spelling required by grammar, each element of a trigram was assigned the stem of a word rather than the word itself. The trigrams presented were ranked by frequency. A summation of frequency of trigram, number of content words in the trigram and number of keywords in the trigram was used as a score for each trigram.

Abridged text was created by computing the key trigrams of a page, as described above, and then using the sentences in which the trigrams with the highest score appeared to provide an abridged version of the page. Abridged text averaged $20 \%$ of the size of the original text.

\section{EVALUATING BROOKESTALK}

\subsection{Keyword evaluation}

If the Web page author has truly encapsulated the meaning of subsections of the page in headings then headings should provide a significantly better indicator of page content than keywords. However headings are often represented as images which provide no speech output, or are eye-catching rather than informative. Here keywords might provide a better summary. The aim of BrookesTalk is to provide flexibility with a range of orientation which can override the vagaries of Web page authoring.

User's perception of the usefulness of the representation was measured by asking them to evaluate the usefulness of describing a Web page using the three different types of summary representations, headings, links/anchors, and keywords.

It is acknowledged that there are differences in the way blind and sighted users listen to and interpret synthetic speech. However sighted subjects were used in this preliminary evaluation because of the possibility of using BrookesTalk over the telephone for Web access [2].

Twenty subject users were shown the different representations for six different Web pages. The pages were chosen to maximise variability. Subjects gave a score between 0 and 5 for each representation. The sum of the scores for each representation, together with the percentage of the total score it represented, was taken to give an indication of its effectiveness, results are shown in Table 2. It can be seen that users perceived that keywords provide a considerable improvement on the use of links to orientate users to Web pages. Headings gave the best score but the score for keywords was not significantly different.

\section{TABLE 2.}

\section{Total Score Percentage of Available Marks}

\begin{tabular}{|l|l|l|}
\hline Headings & 406 & 58.09 \\
\hline Anchors & 313 & 45.24 \\
\hline Keywords & 392 & 55.28 \\
\hline
\end{tabular}

\subsection{Prototype BrookesTalk evaluation}

The BrookesTalk prototype was used by a group of five workers at the Royal National Institute for the Blind (RNIB). They were all technically able. User acceptance was no problem as this group were committed to finding out what software is available for blind people. Our ultimate goal is to develop software for non technical users. However it was felt, for this pilot study, that RNIB workers were best placed to give perspective and comparison with other products.

\subsection{Voices and platform}

BrookesTalk uses different voices for conceptually different parts of a Web page. This was appreciated by most but described as irritating by another. We plan to make different voices optional in the future. 
BrookesTalk runs without any visual display at all and will not run with another browser open. Users felt it would be useful to have the visual equivalent of the spoken page available at the same time so that sighted co-workers could be called in for clarification or work co-operatively with the blind worker.

\subsection{Functionality of BrookesTalk}

Users were observed to rely heavily on one function rather than move between different views, even though they were encouraged to use different views to complement one another. They said they usually know what type of page they are searching for, research work, entertainment, product details etc. You therefore know how useful headings are going to be and make your own decision about the view you want. Surprisingly one user orientated himself by using the links key $90 \%$ of the time, building his conceptual model of the page by looking at what was behind it. He used other browsers for the blind in this way and was reluctant to change even though BrookesTalk had increased functionality.

The abridged page function received most criticism. The trigram analysis easily picked out the wrong trigrams as being significant and important headings were left out of the summary. The algorithm for picking trigrams can be easily influenced by irrelevant words. It was suggested that trigrams should be weighted if they appear in the title or headings.

\subsection{Overall value of BrookesTalk}

Initial evaluation highlighted the potential usefulness of features designed to improve navigation, such as keywords and page summary. Users at RNIB saw the value of using a tool such as BrookesTalk to sort through what they referred to as the 'increasing pile of paper that arrived on their desks'.

Blind users automatically compared BrookesTalk with the browsers they knew e.g. pwWebSpeak(тм) . Initial feedback indicated that the product is fairly accessible for this stage of its development; but some problems exist with accessing links, there is a desire for simultaneous text presentation to allow sighted help, and easier system and voice configuration. Useful aspects included vocal notification of HTML mark-up (i.e. if text is a heading, alt-text etc.) and being able to move between headings and start speech from specified points in the text.

\section{CONCLUSION}

By incorporating usability at such an early stage in the development process the system was thought more likely to meet user needs, with the next step being a more structured approach to obtaining feedback. The pilot exercise was valuable for highlighting basic design problems.

We have now incorporated a visual browser into the system and set aside an area for enlarged text representation of the sentence currently being spoken. Work will go forward in two areas.

We will continue investigation of algorithms for extracting the meaning of a page for the blind employing more natural language processing theory. New work will be started on the use of speech enhancement to visual browsers for the benefit of visually impaired users.

\section{REFERENCES}

1. Zajicek, M. and Powell, C. Building a conceptual model of the World Wide Web for visually impaired users' S. A. Robertson (Ed) Contemporary Ergonomics 1997 (Taylor \& Francis London), $270-275$

2. Zajicek M. and Powell, C. Accessing the World Wide Web by telephone in Proceedings of Designing for our future selves: Human Factors in Telecommunications '97 (Oslo 197), 177-184. 